\title{
Local magnetization profile and geometry magnetization effects in microwires as determined by magneto-optical Kerr effect
}

\author{
Jingfan Ye, Rafael P. del Real, Germán Infante, and Manuel Vázquez \\ Institute of Materials Science of Madrid, CSIC, 28049 Madrid, Spain
}

(Received 15 October 2012; accepted 28 December 2012; published online 23 January 2013)

\begin{abstract}
The local magnetization profile along the length in magnetostrictive Fe-based magnetic microwires has been determined by magneto-optical Kerr effect. The study has been performed in microwires with different geometrical dimensions (i.e., diameter and length). The profiles of remanent magnetization and coercivity remain constant at the middle part for all microwires, whereas significant reduction of net magnetization accompanied by significant change of coercivity is observed when approaching their ends. This local region extends just few tens of micrometer for thin (around $1 \mu \mathrm{m}$ diameter) wires and up to several hundreds of micrometer for thick (around $10 \mu \mathrm{m}$ diameter) wires. That predicts that critical length to observe bistability goes from $50 \mu \mathrm{m}$ to nearly $1 \mathrm{~mm}$ as diameter increases from 1 to $10 \mu \mathrm{m}$. Results are further interpreted considering the local distribution of magnetic charges at the ends which, arising to reduce stray fields, lead in some cases to inverted loops. (C) 2013 American Institute of Physics.
\end{abstract}

[http://dx.doi.org/10.1063/1.4776730]

\section{ROLE OF GEOMETRY IN THE MAGNETIZATION REVERSAL OF MICROWIRES}

The energy balance among magnetic anisotropy terms determines the static distribution of magnetization into magnetic domains as well as the dynamic magnetization process upon application of increasing magnetic field. The case of amorphous magnetic wires is very particular in the sense that only two terms are relevant: (i) the magnetoelastic anisotropy (arising from the coupling between strong mechanical stresses frozen during the preparation and the magnetostriction constant) and (ii) the shape anisotropy (determined by the cylindrical shape itself). In addition, local fluctuations of magnetic anisotropy can arise from intrinsic amorphous structure, as well as local geometry inhomogeneities or imperfections produced during the fabrication process. General information on magnetism of amorphous microwires can be found in several review articles. ${ }^{1-3}$

In the case of Fe-based wires with high positive magnetostriction, the magnetoelastic anisotropy reinforces the shape anisotropy leading to a quite large core single domain structure axially magnetized. In addition, it is generally assumed that closure domain structures form at the ends to diminish stray field energy as well as at the surface with radial magnetization easy axis. Magnetization reversal between two stable magnetic configurations takes place by nucleation and propagation of a single domain wall. These characteristics make bistable microwires a model system where to study basic magnetization processes and also to be used as sensing elements in a number of sensor devices. The present research trends, fundamental and applied, in magnetic microwires have been updated recently. ${ }^{4}$

Full understanding of fine details of magnetization reversal in bistable wires is fundamental to advance in basic and applied research. ${ }^{5}$ The microwire's diameter and length (i.e., the aspect ratio) define the macroscopic demagnetizing field that determines a critical length below which bistability is lost. Reversal under homogeneous axial field spontaneously occurs by depinning of a single domain wall from the closure structure at one end of the wire. Consequently, the determination of local magnetization distribution is essential. First studies on the magnetization profile in amorphous wires were performed years ago from experimental and micromagnetic viewpoints. ${ }^{6-10}$ Measurements were typically performed by fluxmetric induction technique using small secondary coils detecting local magnetization with precision of 1-2 $\mathrm{mm}$ as determined by the pickup width.

Magneto-optical Kerr effect imaging is being successfully employed for the direct observation of magnetic domains ${ }^{11-13}$ in magnetic microwires. Nevertheless, less attention has been paid to obtain complementary information, i.e., hysteresis loops and its parameters. Recently, optimized equipment is enabling to obtain the magnetic response in a local region as small as few micrometers. ${ }^{14}$

The objective of the present study is first to obtain detailed information on the role played by geometry, specifically the diameter and length of microwires, in the magnetization switching; and more concretely, to determine the local distribution of magnetic moments at the region close to the end where magnetization reversal begins. To achieve that, we employ longitudinal magneto-optical Kerr effect with micrometric resolution.

\section{PREPARATION OF MICROWIRES WITH DIFFERENT DIAMETER AND LENGTH}

Glass-coated amorphous microwires have been prepared in our laboratory by quenching and drawing, a rapidsolidification technique which is sometimes known as modified Taylor-Ulitovsky method. ${ }^{15}$ The nominal alloy composition of wires selected for measurements was $\mathrm{Fe}_{75} \mathrm{Si}_{15} \mathrm{~B}_{10}$ that is a typical composition with high magnetostriction constant $(+32 \mathrm{ppm})$ for which magnetic bistability is ensured. This magnetic metallic core with diameter, $d_{m}$, is coated by 

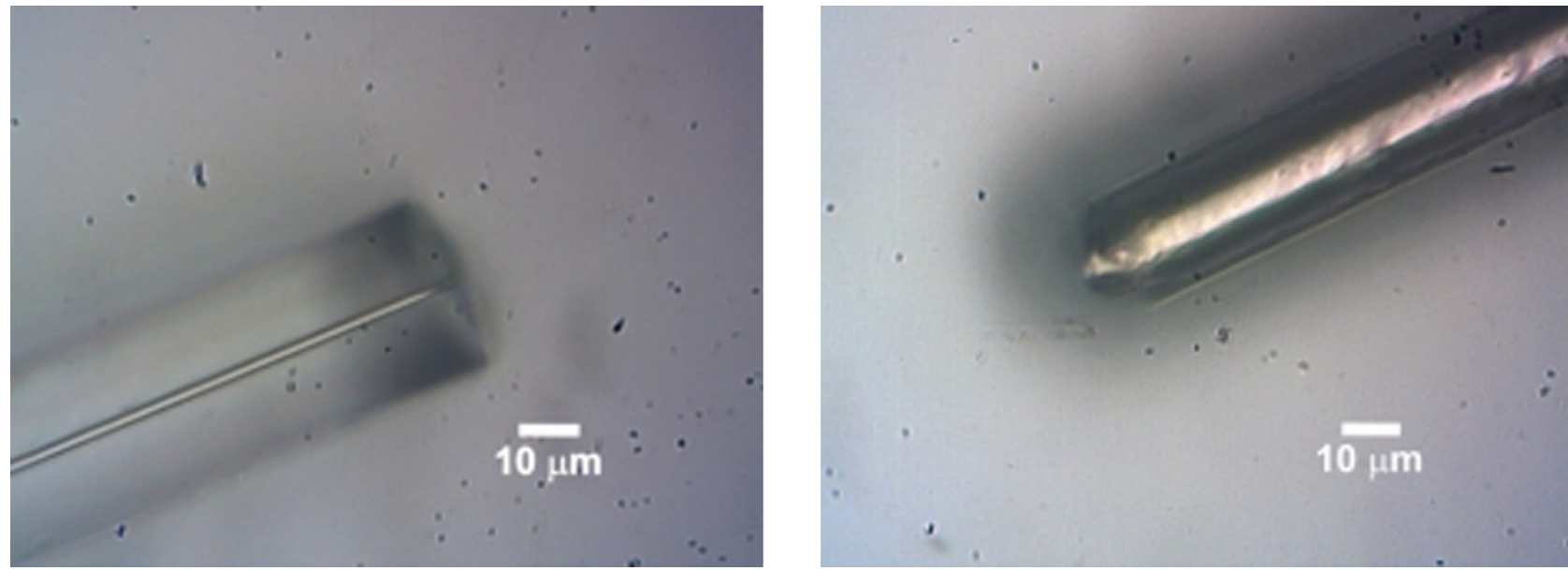

FIG. 1. Optical microscope images of the very end of two glass-coated microwires with metallic diameter $d_{m}=1.5$ and $12 \mu \mathrm{m}$.

insulating protecting Pyrex whose thickness is tailored by suitable choice of fabrication parameters to give a total diameter of the wire, $D_{T}$. The diameter of the metallic nucleus was tuned between 1 and $12 \mu \mathrm{m}$, while the thickness of the Pyrex cover was varied between 4 and $14 \mu \mathrm{m}$. Figure 1 shows the images of the ends of two glass-coated microwires.

Pieces of microwires around $L=9 \mathrm{~mm}$ long were taken for measurements. The extremely careful cutting process (performed with scalpel on wires fixed between two layers of Parafilm) should be emphasized as it is needed to obtain good samples for measuring local effects close to the end of wires. This cutting process was subsequently performed in order to achieve shorter samples (down to around $1 \mathrm{~mm}$ in length) to investigate the influence of the length of wires on the magnetic behavior.

\section{GEOMETRY EFFECTS ON THE MAGNETIC PROPERTIES OF MICROWIRES AS DETERMINED BY VIBRATING SAMPLE MAGNETOMETER}

A first magnetic characterization of microwires with different diameter and length was performed in a Vibrating Sample Magnetometer, VSM, (KLATencor, EV7) under a maximum applied field of $1.5 \mathrm{~T}$. Fig. 2 shows the hysteresis

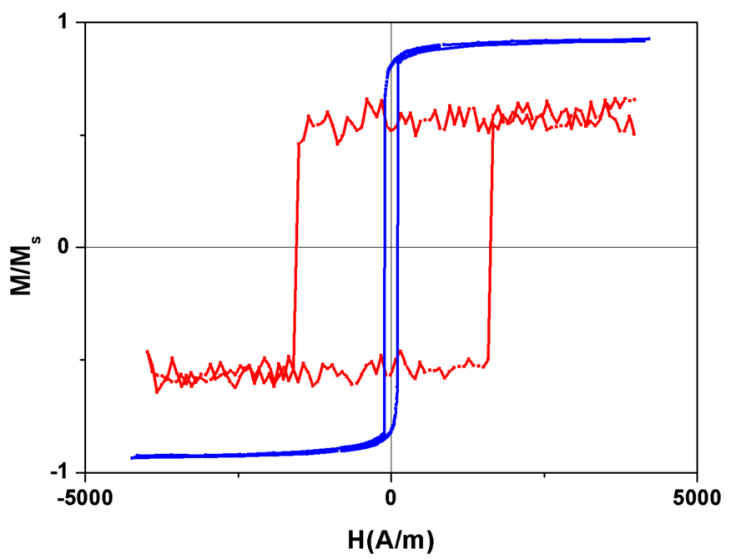

FIG. 2. VSM hysteresis loops for a thin microwire $\left(d_{m}=1.2 \mu \mathrm{m}\right)$ and thick one $\left(d_{m}=12 \mu \mathrm{m}\right)$. In both cases, the length of microwires was $5 \mathrm{~mm}$. loop of two samples with different metallic diameter and nearly constant total diameter: a "thick" sample $\left(d_{m}=12 \mu \mathrm{m}\right.$, $\left.D_{T}=29 \mu \mathrm{m}\right)$ and a "thin" sample $\left(d_{m}=1.5 \mu \mathrm{m}, D_{T}=30 \mu \mathrm{m}\right)$. Two facts make them different, the coercive field, larger for the thin wire, and the fractional remanent magnetization, larger for the thick sample. The magnetically harder magnetic behavior of the microwire with thinner metallic core is a consequence of the stronger mechanical stresses introduced on the magnetic core by the insulating coating as has been previously reported. $^{7,8,14,15}$

Note that from Fig. 2 it can be derived that the thinner wire needs stronger magnetic field to reach magnetic saturation (not shown in the figure). After the large Barkhausen jump, its magnetization increases reversibly up to near saturation at $60 \mathrm{kA} / \mathrm{m}$.

It is also worth to underline that the magnetization process when approaching to the large Barkhausen jump at the switching, $M_{s w}$, from the remanent, $M_{r}$, state is different as well. In the case of the thin sample, this approach is very abrupt, $\left(M_{r}-M_{s w}\right) \approx 0$, that suggests that there is not a significant angular reorientation of spins (including the region close to the ends of the wire) before the depinning and propagation of the single domain wall from one end of the wire. Nevertheless, for the thick sample, the zooming of the region indicates that $\left(M_{r}-M_{s w}\right) \approx 0.16 M_{s}$. That suggests a difference in the formation of the domain wall (i.e., the growing of already nucleated reverse domain) before its depinning.

The influence of geometry can be straightforward observed in Fig. 3 showing the hysteresis loops (only lowfield region is depicted) of thick microwire pieces with decreasing length from 9 down to $1 \mathrm{~mm}$ out of the same original piece $L=9 \mathrm{~mm}$ long. Perfect magnetic bistability (i.e., reversal through a single giant Barkhausen jump, $\Delta M_{B}$ ) is only observed in wires above $3 \mathrm{~mm}$ in length. That critical length to observe bistable behavior is confirmed to decrease as the microwire diameter decreases. For non-bistable loop short microwires stronger field is required to approach magnetic saturation (not shown here). The inset of Fig. 3 shows the evolution of $M_{r} / M_{s}$ and $\Delta M_{B} / M_{s}$ for these samples showing that the smaller the length of the microwire, the smaller the remanent magnetization. 


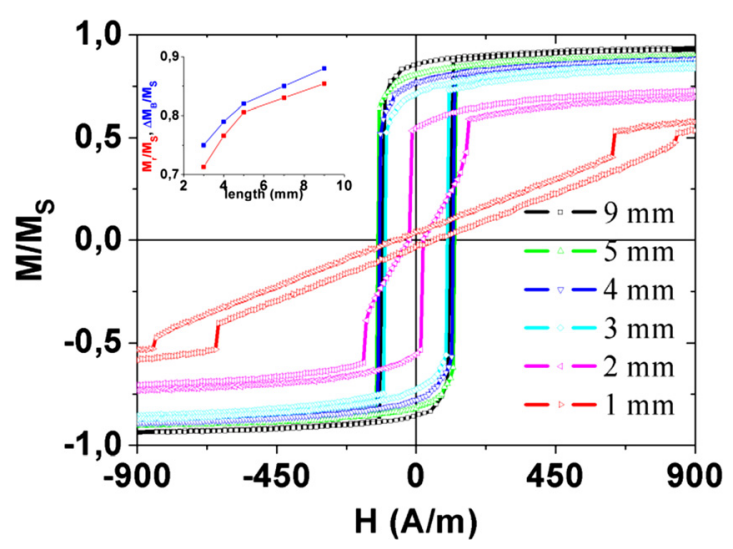

FIG. 3. Hysteresis loops of microwires $\left(d_{m}=12 \mu \mathrm{m}\right)$ with different lengths. Inset depicts the length dependence of the large Barkhausen jump and of the variation remanence to magnetization at the switching.

\section{MAGNETO-OPTICAL KERR EFFECT MEASUREMENTS}

The magnetic behaviour of microwires has been investigated by magneto-optical Kerr effect, MOKE, in NanoMOKE 2 by Durham Magneto Optics Ltd. We take advantage of a small focusing zone, around $3 \mu \mathrm{m}$ diameter, to achieve very local information on the magnetization distribution in the wires. Longitudinal MOKE hysteresis loops were obtained under maximum applied field of $8000 \mathrm{~A} / \mathrm{m}$.

The fractional remanence and coercive or switching field profiles in the whole length are shown in Fig. 4 for thin microwires with $d_{m}=1.5 \mu \mathrm{m}$ and $D_{T}=30 \mu \mathrm{m}$ after successive cutting processes of the same initial piece of microwire with length $L=8 \mathrm{~mm}$. The abscissas logarithmic scale is chosen to emphasize changes close to the ends. We should first underline the sensitivity of the measurement by which we obtain information at the end with micrometric scale resolution. Remanence and coercivity remain roughly unchanged after the cutting processes and they show the same values up to the micrometric region at the end, irrespective of the microwire length. It is really exciting to observe that reduction of both coercivity and remanence is observed only in the region extending around 20 to $30 \mu \mathrm{m}$ from the end. That confirms first that the precise cutting process does not introduce any significant defect or mechanical stress. From the profile of the remanence, we deduce that its reduction at the end is caused by the local redistribution of magnetic moments to diminish the local stray fields that eventually results in a kind of complex closure domains. Moreover, we would anticipate a critical length to observe bistability of the order of double such a distance, that is, just around $50 \mu \mathrm{m}$. Besides that, for these thin microwires, the coercive field always decreases close to the ends, and therefore the surface appears to be magnetically softer.

Fig. 5 depicts the fractional remanence and switching field profiles for a thick microwire $\left(d_{m}=12 \mu \mathrm{m}\right.$ and $\left.D_{T}=22 \mu \mathrm{m}\right)$, after successive cutting processes from an original length $L=8 \mathrm{~mm}$. In this case, we observe that remanence and coercivity take constant values in a broad central region but at the ends where they undergo very significant changes. We should emphasize nevertheless several differences in comparison with thin microwires. First, coercivity takes much smaller values in that central part, indicating a
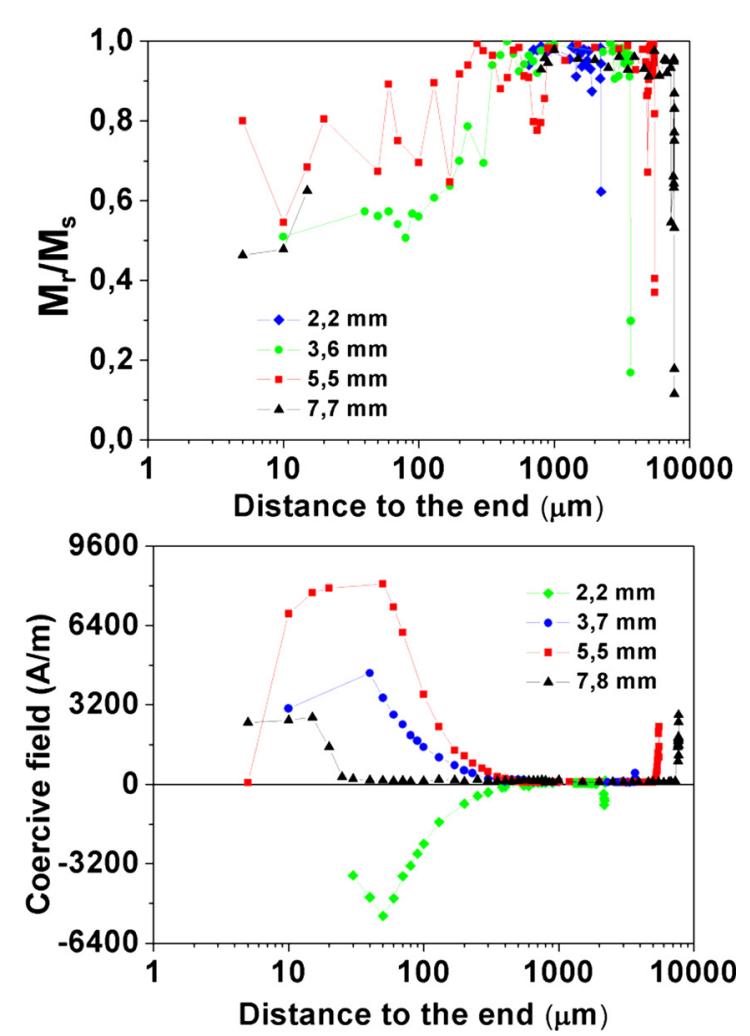

FIG. 5. MOKE fractional remanence and coercivity for thick wires $\left(d_{m}=12 \mu \mathrm{m}\right)$ after successive cutting processes to different lengths.
FIG. 4. MOKE fractional remanence and coercivity for thin wires $\left(d_{m}=1.5 \mu \mathrm{m}\right)$ after successive cutting processes to different lengths.
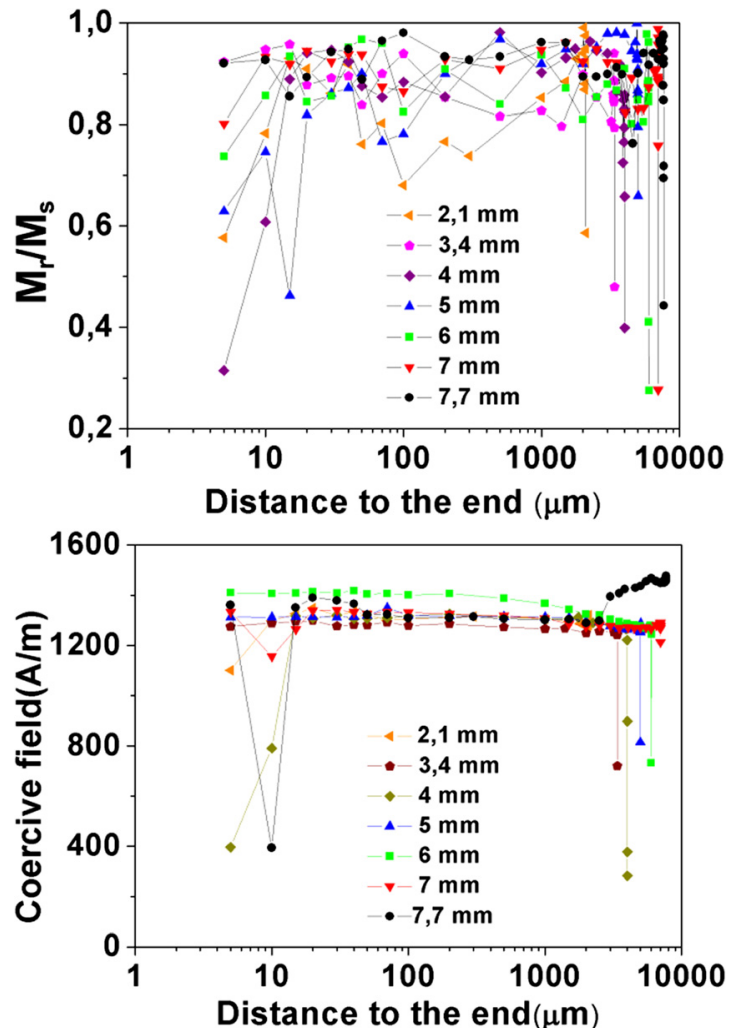
much softer magnetic behaviour as it corresponds to a large metallic diameter and relatively reduced Pyrex thickness in agreement with data shown in Fig. 2. Also, the region close to the ends where remanence but also particularly switching field show very noticeable changes extend till several hundreds of $\mu \mathrm{m}$, one order of magnitude larger than in the case of thin microwires. Looking at these profiles in detail, one observes large local modifications close to the ends that can be summarized in the two following points: (i) there is a large, sometimes huge increase (from 100 to $8000 \mathrm{~A} / \mathrm{m}$ ) of switching field although it tends to decrease at the very end and (ii) local inverted hysteresis loops with "negative" coercivity and remanence are observed for the shorter wire $(2.2 \mathrm{~mm})$ for both ends.

Inverted loops can be observed in more detail in the series of hysteresis loops in Fig. 6 for a microwire with $d_{m}=7 \mu \mathrm{m}, D_{T}=17 \mu \mathrm{m}$, and $L=8 \mathrm{~mm}$. Fig. 6(a) shows the loop at $20 \mu \mathrm{m}$ to the end where coercivity takes a relatively small value of $250 \mathrm{~A} / \mathrm{m}$, while at $70 \mu \mathrm{m}$ (Fig. 6(b)), the hysteresis loop denotes a sudden local magnetic hardening with a "negative" coercivity of $960 \mathrm{~A} / \mathrm{m}$. Fig. 6(c) shows the reverse loop at $190 \mu \mathrm{m}$ distance with coercivity of $-120 \mathrm{~A} / \mathrm{m}$. From $200 \mu \mathrm{m}$ and further towards the middle of the wire, the loop becomes standard and coercivity takes the above mentioned value of about $80 \mathrm{~A} / \mathrm{m}$ (Fig. 6(d) shows the hysteresis loop at $4800 \mu \mathrm{m}$ distance from the end).

This type of observed inverted loops is sometimes observed in selected thin films. ${ }^{16-22}$ To understand it, we should consider that the measured loop actually represents magnetization against applied magnetic field, where the presence of additional field component, particularly local demagnetizing fields, is not taken into account. In our present case of thick wires, the local inverted loops in the region, close but not at the very end of the microwires, denote the existence of a significant local redistribution of magnetic moments. ${ }^{10}$
From the observed extent in Fig. 5 of the region with significant change in remanence and coercivity measured by MOKE, we can deduce a critical length for bistability of around $1 \mathrm{~mm}$ for thick wires, that is, double of the closure domains length. That agrees with VSM data in Fig. 3 if we assume that the difference between the saturation magnetization and the remanence, $\left(M_{s}-M_{r}\right)$, arises from the presence of the closure domain structure. In that case, the length of the closure domains $L_{C D}$ is given by

$$
L_{C D}=\frac{L}{2}\left(1-\frac{M_{r}}{M_{s}}\right) .
$$

From results in Fig. 3, we obtain that these domains extend over $L_{C D}=0.66,0.59,0.48,0.47$, and $0.43 \mathrm{~mm}$ from the end for total microwire length of $9,7,5,4$, and $3 \mathrm{~mm}$, respectively.

\section{DISCUSSION}

From MOKE results, we can derive that at the very end of microwires, there is a micromagnetic redistribution of magnetic moments into a kind of closure domains resulting in a decreased remanence, while local coercivity differs from that at the middle part of the wire. That redistribution of magnetic charges should be understood to form in order to reduce the total magnetostatic energy. That redistribution affects a significant region at the surface that extends say 50 and $800 \mu \mathrm{m}$ for thin and thick wires, respectively.

The very fact that coercivity depends on the local position indicates that a nucleation/propagation reversal mechanism should be considered: a reverse cluster domain nucleates at the end facilitated by the presence of redistributed charges already at remanence. A single domain wall propagates after its depinning from such closure structure. ${ }^{5}$ Coercivity is determined by domain wall pinning or nucleation hardening mechanism. ${ }^{23,24}$ A general expression for the
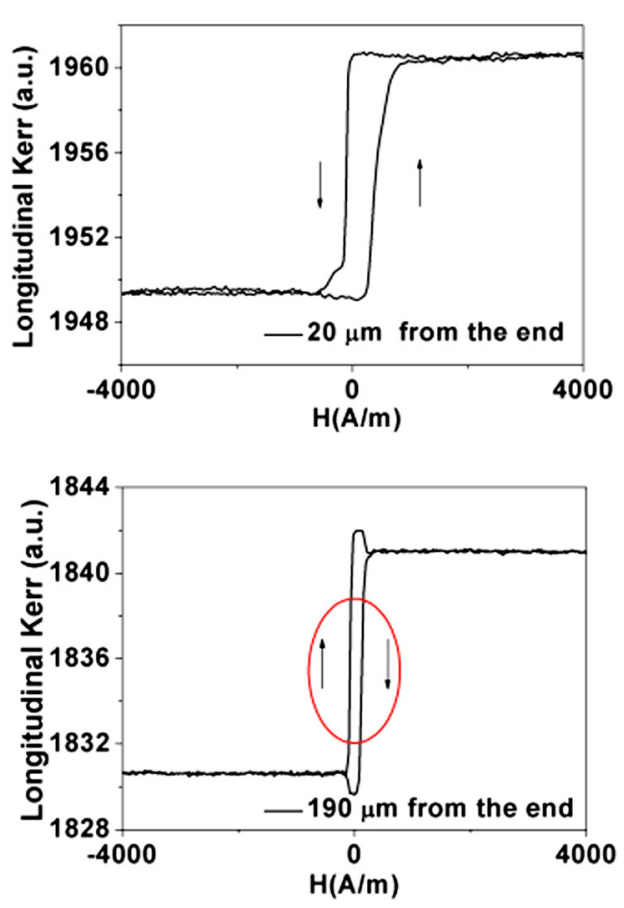
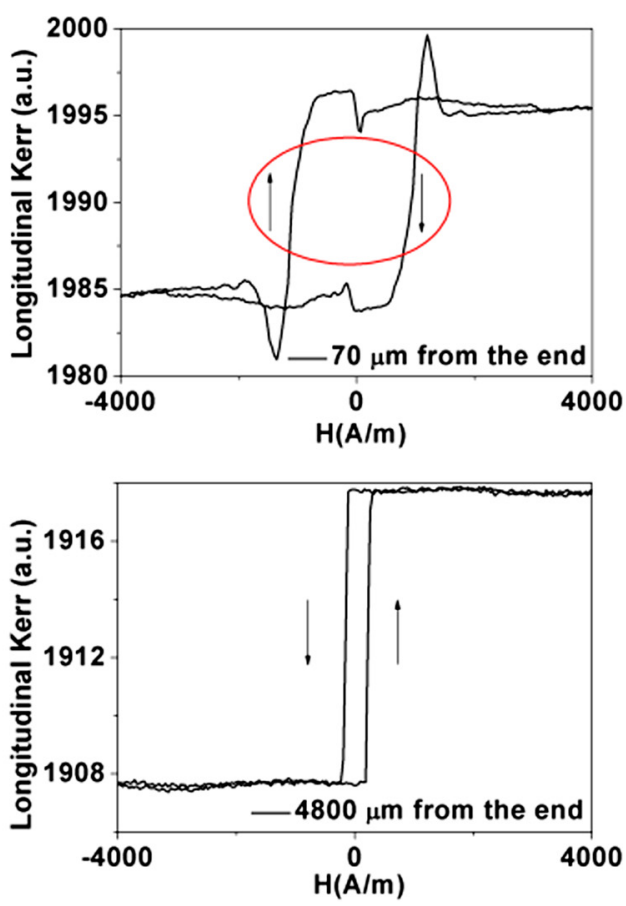

FIG. 6. MOKE hysteresis loops as a function of the distance to the "thick" $\left(d_{m}=7 \mu \mathrm{m}\right)$ wire end. Arrows are included to emphasize the inverse sense of the loops. 
dependence of local coercivity, $H_{c}$, on geometric characteristics of amorphous microwires can be given as

$$
H_{c}(S, L, x)=\alpha \frac{3 \lambda \Omega(s)}{2 \mu_{0} M_{s}} \pm H_{D e m}(\sigma(x), L, x),
$$

where $x$ is the distance to the end of the microwire. The first term at the right hand of Eq. (2) denotes a magnetoelastic anisotropy field contribution which arises from the Zeeman energy needed to nucleate a reverse nucleus including the energy to form the corresponding wall (the $\alpha$ parameter is determined by the wall shape and the temperature activation energy term). Note that the saturation magnetization, $\mu_{0} M_{s}$, and the magnetostriction, $\lambda$, are determined exclusively by the alloy composition, while the internal stress, $\Omega$, depends on the ratio, $S$, between the total, $D_{T}$, and the metallic diameter, $d_{m}$ $\left(S=D_{T} / d_{m}\right)^{2}$. The second term at the right hand of Eq. (1) denotes the local demagnetizing field, $H_{D e m}$, resulting from the particular distribution of magnetic charges, $\sigma(x)$, at both ends of the microwire. Note that in the present case, the expression of $H_{\text {Dem }}$ is more complex than in saturated cylinder. ${ }^{25}$

The local coercive field measured in the central part of microwires, $H_{c}^{c e n t r}$, does not change irrespective of the length for both thin and thick microwires as observed in Figures 4 and 5. This indicates that the net magnetic charge, $\langle\sigma(x)\rangle$, resulting from the presence of the closure domains is largely balanced and that the only significant contribution to coercivity comes from the magnetoelastic anisotropy field. The geometry dependence of stress, $\Omega(S)$, treated in several previous works, ${ }^{2,15,26}$ confirms the results obtained in Figures 2, 4 , and 5, that is, the coercive field at the central part of thin wires is one order of magnitude larger than for thick wires.

On the contrary, at the ends of the microwires, the contribution of the local distribution of magnetic charges is critical. This local demagnetizing effect is very sensitive to local defects and inhomogeneities that in turn enable the nucleation of reverse domain. However, the influence of the demagnetizing field is different for thick and thin microwires when related to both the geometry and the magnetoelastic anisotropy.

For thin wires, coercive field decreases at the very ends as a consequence of the strong local demagnetizing contribution in Eq. (2). For the thick wires, the end region is magnetically harder than at the centre. It denotes not only a strong local demagnetizing field but also that charges may distribute in a different manner giving rise to positive or negative contribution (i.e., observe the positive and negative coercivity values in Fig. 5), and eventually to the appearance sometimes of inverted loops (Fig. 6).

This type of inverted hysteresis loops were micromagnetically proposed ${ }^{16,17}$ and experimentally observed in various systems mainly consisting of layered structures with well-defined interfaces between layers. ${ }^{18-22}$ Such unusual loops are interpreted considering either magnetostatic or exchange interaction between layers with non-identical magnetic phases (i.e., both having uniaxial in plane anisotropy or even ferro/antiferro character). In the present case, inverted loops are a consequence of the strong local demagnetizing fields. Plotted magnetic field in Fig. 6 denotes the applied field while local magnetic moments are actually undergone to applied plus local field so that, total field may reach positive or negative field. It is precisely in that local region where we should assume that reverse magnetic moments nucleate as first clusters for magnetization reversal giving rise to the subsequently propagating domain wall. Further into the centre of the microwire, the local demagnetizing field is negligible and the effective field equates the applied field.

Present data allow us to reach some additional implications regarding the assumed core/shell domain structure model. The radial profile of the domain structure in magnetostrictive Fe-based glass-coated microwires is commonly accepted to present a core axially magnetized and an external shell with nearly radial magnetization distribution. ${ }^{3}$ In fact, MOKE hysteresis loops should reflect the presence of such radial region since it informs of the magnetic response at the microwire surface. The penetration depth, $\delta$, of the MOKE loops has been calculated to be $\delta=(\pi f \mu \sigma)^{-1 / 2} \approx 32 \mathrm{~nm}$ (with the frequency of light $f=4.74 \times 10^{14} \mathrm{~Hz}$, the permittivity $\mu=\mu_{0} \mu_{r} \approx \mu_{0}$, and the conductivity $\left.\sigma=5.18 \times 10^{5}(\mathrm{~m} \Omega)^{-1}\right)$. Nevertheless, surface (MOKE) and bulk (VSM) hysteresis loops at the middle part of wires show quite similar square shape and coercivity values. Such similarity leads one to wonder about the accepted view of the external region with radial domain structure. This region, if existing at all, should require some modification.

Moreover, slight differences are observed when comparing MOKE and VSM data. For example, MOKE and VSM coercivity values are, respectively, $1264 \mathrm{~A} / \mathrm{m}$ and $1376 \mathrm{~A} / \mathrm{m}$ for $d_{m}=1.5 \mu \mathrm{m}$ and $D_{T}=29 \mu \mathrm{m}, 1480$ and $1760 \mathrm{~A} / \mathrm{m}$ for $d_{m}=2.2 \mu \mathrm{m}$ and $D_{T}=10 \mu \mathrm{m}$, and $448 \mathrm{Oe}$ and $584 \mathrm{~A} / \mathrm{m}$ for $d_{m}=6 \mu \mathrm{m}$ and $D_{T}=16 \mu \mathrm{m}$ microwires. The slightly smaller MOKE coercivity may suggest that magnetization reversal should begin at the region close to the magnetic wire surface where more dense magnetic charges likely concentrate.

Finally, and regarding VSM data for thick wires, the difference between saturation and remanence, $\left(M_{s}-M_{r}\right)$, increases as the length of the wire is reduced (see Fig. 3). Such increase is coherent with the increase in fractional volume of closure-like domains at the ends of the wire. In turn, the fractional remanence for long enough thin wires is around $0.50 M_{s}$ (see Fig. 2). Its difference from saturation magnetization is too high to be ascribed just to the presence of closure-like domains or to the outer shell (ruled out by MOKE measurements as shown above). To account for that, we should assume that magnetization actually exhibits a helical, rather than pure longitudinal, configuration inside the large central domain with a significant angle from the axis of the wire. $^{27}$

\section{CONCLUSIONS}

Present results confirm the relevant role of geometric effects (i.e., metallic diameter) in the reversal process. The analysis of measured local MOKE hysteresis loops and its parameters in glass-coated Fe-based magnetostrictive microwires allows us to reach the following conclusions:

1. Regardless of the microwire diameter, MOKE hysteresis loops in the middle part exhibit bistable behavior even 
though the penetration is only at the nanoscale. It suggests that the core central magnetic domain fills the whole cross section of the microwire, and therefore, the outer shell with radial magnetization should be seemingly dismissed.

2. Thin wires (around $1 \mu \mathrm{m}$ metallic diameter) behave as magnetically harder, particularly at the middle part, in comparison with thick wires. That is ascribed to the stronger stresses induced by the Pyrex coating.

In turn, the local demagnetizing fields play very important role to decrease local coercivity at the ends. The local redistribution of magnetic moments extends up to around $20 \mu \mathrm{m}$ from the end, from which a 40 to $50 \mu \mathrm{m}$ critical length to observe bistability. Besides, the magnetization in the central domain is proposed to be helically oriented.

3. Thick wires (around $10 \mu \mathrm{m}$ metallic diameter) are magnetically softer at the middle part because of the comparatively weaker mechanical stress induced by the Pyrex coating.

At a region close to the end extending up to around $500 \mu \mathrm{m}$, magnetic moments distribute in a complex manner producing strong local demagnetizing fields that result either in high coercivity or alternatively inverted loops. In this case, the predicted critical length to observe bistability is of around $1 \mathrm{~mm}$, which agrees well with VSM measurements.

The enhanced coercivity of thick wires at the ends suggests that, for low applied field, magnetization reverses through the propagation of a domain wall along the main middle region of the wire without significant change at the ends.

\section{ACKNOWLEDGMENTS}

Jingfan Ye acknowledges the support of the German DAAD under the programme "Research Internships in Science and Engineering (RISE-2011)." Authors wish to thank Dr. G. A. Badini-Confalonieri and A. Jimenez for support in preparation of samples. The work has been supported by the Spanish Ministry of Economy and Competitiviness under projects PLE2009-0057 and MAT2010-20798-C05-01.
${ }^{1}$ M. Vázquez, "Advanced magnetic microwires," in Handbook of Magnetism and Advanced Magnetic Materials, edited by H. Kronmüller and S. S. K. Parkin (Wiley, Chichester, England, 2007), Vol. 4, p. 2193.

${ }^{2}$ A. Zhukov and V. Zhukova, Magnetic Properties and Applications of Ferromagnetic Microwires with Amorphous and Nanocrystalline Structure (Nova Science, Hauppauge, NY, 2009), Vol. 162, p. 11788.

${ }^{3}$ H. Chiriac and T. A. Ovari, Prog. Mater. Sci. 40, 333 (1996).

${ }^{4}$ M. Vazquez, H. Chiriac, A. Zhukov, L. Panina and T. Uchiyama, Phys. Status Solidi A 208, 493 (2011).

${ }^{5}$ M. Vazquez, G. A. Basheed, G. Infante, and R. P. del Real, Phys. Rev. Lett. 108, 037201 (2012).

${ }^{6}$ D. X. Chen, C. Gomez-Polo, and M. Vazquez, J. Magn. Magn. Mater. 124, 262 (1993).

${ }^{7}$ A. P. Zhukov, M. Vazquez, J. Velazquez, H. Chiriac, and V. Larin, J. Magn. Magn. Mater. 151, 132 (1995).

${ }^{8}$ V. Zhukova, N. A. Usov, A. Zhukov, and J. Gonzalez, Phys. Rev. B 65, 134407 (2002).

${ }^{9}$ S. Gudoshnikov, N. Usov, A. Zhukov, J. Gonzalez, and P. Palvanov, J. Magn. Magn. Mater. 316, 188 (2007).

${ }^{10}$ I. Betancourt, G. Hrkac, and T. Schrefl, J. Appl. Phys. 109, 013902 (2011).

${ }^{11}$ A. Chizhik, R. Varga, A. Zhukov, J. Gonzalez, and J. M. Blanco, J. Appl. Phys. 103, 07E707 (2008).

${ }^{12}$ A. Chizhik, A. Zhukov, J. Gonzalez, and J. M. Blanco, J. Appl. Phys. 105, 123911 (2009).

${ }^{13}$ H. Chiriac, J. Yamasaki, T. A. Ovari, and M. Takajo, IEEE Trans. Magn. 35, 3901 (1999).

${ }^{14}$ H. Chiriac and T. A. Ovari, J. Magn. Magn. Mater. 323, 2929 (2011).

${ }^{15}$ V. S. Larin, A. V. Torcunov, A. Zhukov, J. González, M. Vázquez, and L. Panina, J. Magn. Mater. 249, 39 (2002).

${ }^{16}$ A. S. Arrot, in Nanomagnetism, edited by A. Hernando (Kluwer, Dordrecht, 1993), p. 73

${ }^{17}$ A. Aharoni, J. Appl. Phys. 76, 6977 (1994).

${ }^{18}$ M. Tejedor, H. Rubio, and A. Fernandez, Appl. Phys. Lett. 55, 1920 (1989).

${ }^{19}$ M. Cougo dos Santos, J. Geshev, J. E. Schmidt, S. R. Teixeira, and L. G. Pereira, Phys. Rev. B 61, 1311 (2000).

${ }^{20}$ K. Takanashi, H. Kurokawa, and H. Fujimori, Appl. Phys. Lett. 63, 1585 (1993).

${ }^{21}$ E. E. Shalyguina, V. V. Molokanov, M. A. Komarova, V. A. Melnikov, and N. M. Abrosimova, J. Magn. Magn. Mater. 290-291, 1438 (2005).

${ }^{22}$ P. W. Haycock, M. F. Chioncel, and J. Shah, J. Magn. Magn. Mater. 242-245, 1057 (2002).

${ }^{23} \mathrm{H}$. Kronmüller and M. Fähnle, Micromagnetism and the Miscrostructure of Ferromagnetic Solids (Cambridge University Press, Cambridge, 2003), p. 90.

${ }^{24} \mathrm{G}$. Bertotti, Hysteresis in Magnetism (Academic, London, 1998), p. 357.

${ }^{25}$ D.-X. Chen, J. Appl. Phys. 89, 3413 (2001).

${ }^{26}$ S. A. Baranov, V. N. Berzhanskii, and S. K. Zotov, Fiz. Met. Metalloved. 67, 73 (1989).

${ }^{27}$ H. Chiriac, T. A. Ovari, S. G. Marinescu, and V. Nagacevschi, IEEE Trans. Magn. 32(5), 4754 (1996). 